Original Research

\title{
Determination of Pollution and Heavy Metal Fractions in Golden Horn Sediment Sludge (Istanbul, Turkey)
}

\author{
Gurdal Kanat $^{1}$, Bahar Ikizoglu${ }^{1}$, Gokhan Onder Erguven ${ }^{2 *}$, Berivan Akgun ${ }^{1}$ \\ ${ }^{1}$ Yildiz Technical University, Faculty of Civil Engineering, Department of Environmental Engineering, \\ Istanbul, Turkey \\ ${ }^{2}$ Munzur University, Faculty of Engineering, Department of Environmental Engineering, \\ Tunceli, Turkey
}

Received: 22 September 2017

Accepted: 25 November 2017

\begin{abstract}
In our study we studied pollution and heavy metal fractions in the Golden Horn (Istanbul, Turkey). This area has been exposed to domestic and industrial pollution for many years. Concentrations of $\mathrm{Zn}$, $\mathrm{Cr}, \mathrm{Cu}, \mathrm{Pb}, \mathrm{Cd}$, and $\mathrm{Ni}$ heavy metals were determined in the study that was conducted to monitor the current state of pollution and changing conditions in the polluted estuary. Mean heavy metal concentrations were found as: $\mathrm{Zn} 70-260, \mathrm{Cr} 26-110, \mathrm{Cu} 70-135, \mathrm{~Pb} 8-50, \mathrm{Cd} 0.1-2$, and $\mathrm{Ni}$ 10-38 mg/ kg. Results of the analysis showed that metal concentrations were usually low, indicating that the sediment was not too polluted in the present case. The range of ratios, different fractions of metal concentrations, and total metal concentrations in the samples were critical for potential toxicity and mobility of metals. Results of the analysis showed that heavy metal fractions in the sediment were usually found in residual forms. The values obtained in the study were also compared with the literature. It was found that surface sediments in the Golden Horn were less polluted than other areas. Establishment of sewer collectors and wastewater treatment plants has improved the heavy metal levels in the sediment.
\end{abstract}

Keywords: heavy metals, wastewater, estuaries, pollution, metal fractions

\section{Introduction}

The Golden Horn (Halic in Turkish) is a $7.5 \mathrm{~km}$ long marine extension into the land in Istanbul at the entrance to the Marmara Sea. Urban and industrial wastes have been discharged to the Golden Horn for many years. The area is also filled with materials transported by

*e-mail: gokhanondererguven@gmail.com
Alibeykoy and Kagithane streams. There were several factories, slaughterhouses, shipyards, and wholesale vegetable and fruit markets in the vicinity of the Golden Horn, and there have been no treatment facilities for many years, while all the facilities discharge their waste into the Golden Horn. As the business opportunities increased in the district, urban sprawl around the Golden Horn increased as well. The diligent work conducted in recent years has partially limited the above-mentioned pollution. 
Pollution sources in the Golden Horn include not only domestic and industrial wastewater but also solid wastes, garbage, and the dreg material transported by Alibeykoy and Kagithane streams, erosion, ballast water drained from ships, and polluted sediment sludge. Emissions along roads also promote an increase in the concentration of pollutants [1]. Approximately $50,000 \mathrm{~m}^{3}$ of water and $59,000 \mathrm{~m}^{3}$ of sediment are transferred to the Golden Horn from Alibeykoy and Kagithane stream basins, which have a drainage area of $380 \mathrm{~km}^{2}$. In the Golden Horn, where the sedimentation is about $3.5 \mathrm{~cm} /$ year, inorganic matter, nutrients, heavy metals, and hydrocarbons have accumulated in the benthic sediment [2-3].

Because the municipalities had inadequate solid waste collection facilities in the early 1980 s, several organizations around the Golden Horn removed their trash by directly disposing of it into the water. Due to erosion, sediments and residues accumulated in the Golden Horn caused an annual depth reduction of 6-10 $\mathrm{cm}$ and filled the Golden Horn basin bed, in addition to being a source of pollutants. Furthermore, the waste that has been thrown into the sea at ship dismantling facilities and the water leakages from garbage disposal sites have also been transferred to the Golden Horn and increased pollution [4].

Although dissolved heavy metals are not present in sediments, they could return to the water column from the sediment via physical and biological processes. The accumulation of pollutants in sediments negatively affects the natural life and organisms in the environment and causes environmental problems. Sediment analysis is quite important in understanding the distribution and transfer of toxic substances. Most common heavy metals that could be conducted in sediment analysis are $\mathrm{Zn}, \mathrm{Cr}$, $\mathrm{Cu}, \mathrm{Pb}, \mathrm{Cd}, \mathrm{Fe}$, and $\mathrm{Ni}[5]$.

The main objective of the present study was to determine the current pollution level in the Golden Horn,

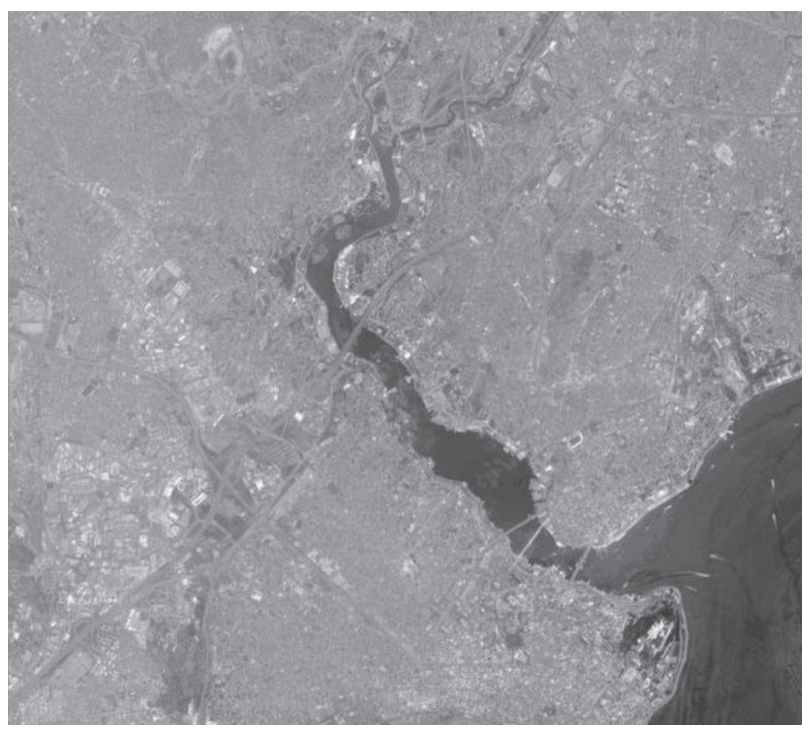

Fig. 1. Satelite view of the Golden Horn. which was exposed to domestic and industrial pollution for several years, and to determine how the current ecological structure of the area, which was attempted to be cleaned at large cost, was affected by the treatment activities.

The present study aimed to analyze various fractions of metals that are significant in sediment pollution. In the analyses, initially the basic parameters such as chemical oxygen demand (COD), total organic carbon (TOC), total solid matter(TSM), total phosphorus (TP), suspended solid material (SSM), total volatile solid matter (TVSM), and total Kjeldahl nitrogen (TKN) were measured, and then the total heavy metal concentrations and concentration values for various fractions were determined.

\section{Material and Methods}

\section{Properties of the Golden Horn}

Topographic structure of the Golden Horn includes the coastline, hills and ridges, slopes and terraces. On both sides of the Golden Horn there is a flat coastline a few centimeters above sea level and $150 \mathrm{~m}$ wide. The most important elements in the Golden Horn region are the hills and extensions, which range between 50 to $130 \mathrm{~m}$ and are located on both slopes [4, 6] (Fig. 1).

\section{Experimental Studies}

In the study, physical and chemical analyses were conducted on the Golden Horn sediment samples and heavy metal pollution and fractions were determined, and existing pollution and changes were monitored. The sediment samples used in the experiments were obtained from the bottom sludge in the Golden Horn-Sutluce region. Currently, Istanbul Metropolitan Municipality Directorate of Maritime Services is conducting dredging on the estuary sediment sludge to remove erosion and polluting materials in this area. Sludge samples were obtained from this sediment. Received samples were delivered to the laboratory as soon as possible under adequate conditions and kept in the refrigerator at $+4^{\circ} \mathrm{C}$. COD, TOC, TSM, TP, SSM, TVSM, and TKN analyses were conducted to determine sediment pollution according to Standard Methods [7].

The total heavy metal concentration and the concentrations of various fractions were processed based on the principles depicted in Standard Methods with disintegration techniques and analyzed. The $\mathrm{Zn}, \mathrm{Cr}, \mathrm{Ni}$, $\mathrm{Cu}, \mathrm{Fe}$, and $\mathrm{Pb}$ contents in samples obtained from the bottom sludge of the estuary were measured with an atomic absorption spectrometer (AAS) and inductive coupled plasma optical emission spectrometer (ICP-OES) to determine the total heavy metal content and heavy metal fractions. Before chemical analyses, the sludge samples were dried at $103^{\circ} \mathrm{C}$ for 24 hours in a Nuve FN 500 -type incubator. The analysis method is summarized below. 
Table 1. Mean heavy metal concentrations ( $\mathrm{mg} / \mathrm{kg}$ dry weight).

\begin{tabular}{|c|c|c|}
\hline Element & Summer & Winter \\
\hline $\mathrm{Zn}$ & $70-200$ & $85-260$ \\
\hline $\mathrm{Cr}$ & $26-90$ & $35-110$ \\
\hline $\mathrm{Cu}$ & $80-135$ & $70-120$ \\
\hline $\mathrm{Pb}$ & $8-50$ & $12-50$ \\
\hline $\mathrm{Cd}$ & $0.1-2$ & $0.2-2$ \\
\hline $\mathrm{Ni}$ & $10-35$ & $12-38$ \\
\hline
\end{tabular}

The sediment samples were exposed to the processes of drying, pulverization, sieving, weighing, and disintegration with acid in the laboratory. For metal measurement, 1-2 grams of each homogeneous sample were weighed on a precision scale and placed in beakers.

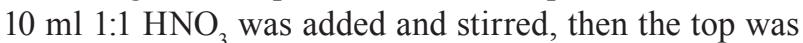
covered with watch glass and heated on a hot plate at $95^{\circ} \mathrm{C}$. After the sample was cooled, $5 \mathrm{ml}$ concentrated $\mathrm{HNO}_{3}$ was added and the beaker was covered with watch glass and kept for 30 minutes for reflux. After this step was completed, the sample was cooled and $2 \mathrm{ml}$ distilled water and $3 \mathrm{ml} 30 \% \mathrm{H}_{2} \mathrm{O}_{2}$ were added. The beaker was covered with watch glass and placed on a hot-plate to initiate the peroxide reaction. After the preliminary treatment, the solutions were filtered through a filter with a pore diameter of $0.45 \mu \mathrm{m}$ and became ready for analysis.
Although sequential extraction of metals requires more time for analysis, it provides important information on their origin, mode of formation, biochemical and physico-chemical use in the aquatic environment, mobile status or their transmission in the aquatic environment.

In the scope of the study, physicochemical properties and heavy metal content of bottom sludge were determined using the samples obtained from the sediment layer. The obtained results were compared with the range of values used to determine sediment quality in the literature.

\section{Results and Discussion}

COD was determined by the closed reflux titrimetric method and it was found to average as $2,650 \mathrm{mg} / \mathrm{L}$ in the samples analyzed at various periods. SSM and VSSM were determined as $0.26 \mathrm{mg} / \mathrm{L}$ and $0.016 \mathrm{mg} / \mathrm{L}$, total solid matter (TSM) was $0.5 \mathrm{~g} / \mathrm{g}$, and TVSM was determined as $0.078 \mathrm{~g} / \mathrm{g}$, respectively. TP was found as $1,630 \mathrm{mg} / \mathrm{kg}$ and finally, TKN was $3.7 \mathrm{mg} / \mathrm{g}$.

In the study, heavy metal concentrations $(\mathrm{Zn}, \mathrm{Cr}, \mathrm{Cu}$, $\mathrm{Pb}, \mathrm{Cd}$, and $\mathrm{Ni}$ ) that originate from human activity were determined. As shown in Table 1, concentrations were usually low and these values indicated that the sediment was not too polluted presently.

The values determined in the literature that reflect the correlation between water ecosystem quality and the metal concentrations showed that certain descriptions were determined. In these studies, the adequate level

Table 2. Threshold effect concentrations (TEC) values that show sediment quality in clear water ecosystem.

\begin{tabular}{|c|c|c|c|c|c|c|}
\hline \multirow{2}{*}{$\begin{array}{c}\text { Element } \\
(\mathrm{mg} / \mathrm{kg} \text { dry weight })\end{array}$} & \multicolumn{6}{|c|}{ Threshold effect concentrations } \\
\hline & TEL & LEL & MET & ERL & TEL-HA28 & TEC \\
\hline $\mathrm{Zn}$ & 123 & 120 & 150 & 120 & 98 & 121 \\
\hline $\mathrm{Cr}$ & 37.3 & 26 & 55 & 80 & 36 & 43.4 \\
\hline $\mathrm{Cu}$ & 35.7 & 16 & 28 & 70 & 28 & 34.6 \\
\hline $\mathrm{Pb}$ & 35 & 31 & 42 & 35 & 37 & 35.8 \\
\hline $\mathrm{Cd}$ & 0.6 & 0.6 & 0.9 & 5 & 0.58 & 0.99 \\
\hline $\mathrm{Ni}$ & 18 & 16 & 35 & 30 & 20 & 22.7 \\
\hline
\end{tabular}

Table 3. Probable effect concentrations (PEC) values in clear water ecosystem (mg/kg dry weight).

\begin{tabular}{|c|c|c|c|c|c|c|}
\hline \multirow{2}{*}{$\begin{array}{c}\text { Element } \\
(\mathrm{mg} / \mathrm{kg} \text { dry weight })\end{array}$} & \multicolumn{6}{|c|}{ Probable effect concentrations } \\
\cline { 2 - 8 } & PEL & SEL & TET & ERM & PEL-HA28 & PEC \\
\hline $\mathrm{Zn}$ & 315 & 820 & 540 & 270 & 540 & 459 \\
\hline $\mathrm{Cr}$ & 90 & 110 & 100 & 154 & 120 & 111 \\
\hline $\mathrm{Cu}$ & 197 & 110 & 86 & 390 & 100 & 149 \\
\hline $\mathrm{Pb}$ & 91.3 & 250 & 170 & 110 & 82 & 128 \\
\hline $\mathrm{Cd}$ & 3.53 & 10 & 3 & 9 & 3.2 & 4.98 \\
\hline $\mathrm{Ni}$ & 36 & 75 & 61 & 50 & 33 & 48.6 \\
\hline
\end{tabular}


Kanat G., et al.

\begin{tabular}{|c|c|c|c|c|c|c|c|c|}
\hline \multirow{3}{*}{ 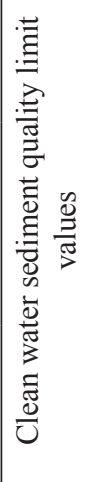 } & 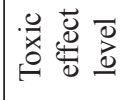 & 1 & ' & $\stackrel{\ominus}{\wedge}$ & $\stackrel{8}{\circ}$ & $\stackrel{\circ}{\circ}$ & ' & ' \\
\hline & 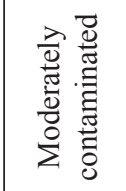 & 1 & 1 & $\begin{array}{l}\stackrel{0}{=} \\
\underline{6} \\
-1\end{array}$ & $\begin{array}{l}8 \\
\stackrel{1}{1} \\
\dot{n}\end{array}$ & $\frac{0}{0}$ & ' & \\
\hline & 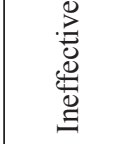 & ' & ' & $\stackrel{\circlearrowright}{v}$ & $\begin{array}{l}0 \\
\text { v }\end{array}$ & $\stackrel{\leftrightarrow}{\dot{V}}$ & ' & 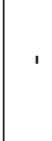 \\
\hline \multirow{2}{*}{ 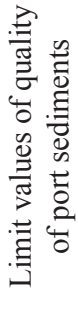 } & 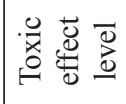 & 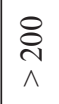 & $\stackrel{n}{\wedge}$ & $\stackrel{\circ}{\wedge}$ & $\begin{array}{l}8 \\
\wedge\end{array}$ & $\stackrel{\circ}{\circ}$ & $\stackrel{n}{i}$ & 员 \\
\hline & 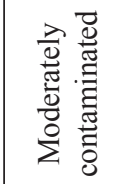 & 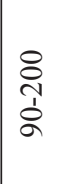 & \begin{tabular}{l}
$n$ \\
$\hat{1}$ \\
\multirow{2}{*}{}
\end{tabular} & $\begin{array}{l}0 \\
\text { ñ } \\
n\end{array}$ & $\begin{array}{l}8 \\
\dot{1} \\
\dot{9}\end{array}$ & ' & 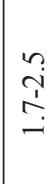 & in \\
\hline \multirow{3}{*}{ 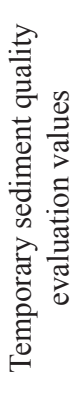 } & 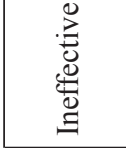 & 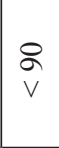 & 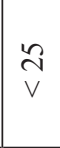 & 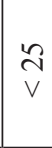 & $\stackrel{q}{q}$ & ' & $\stackrel{\text { I }}{\mathrm{v}}$ & 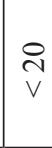 \\
\hline & 总 & $\begin{array}{l}\infty \\
\dot{+} \\
m\end{array}$ & 8 & ๑ & $\frac{m}{\alpha}$ & $\hat{n}$ & ' & iे \\
\hline & 藏 & $\overrightarrow{\tilde{d}}$ & $\stackrel{?}{\stackrel{n}{m}}$ & $\hat{n}$ & $\ddot{m}$ & हैं & , & $\stackrel{\infty}{-1}$ \\
\hline \multirow{3}{*}{ 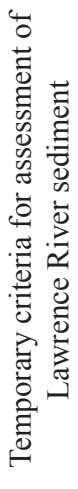 } & 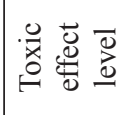 & 早 & \& & $\triangleright$ & 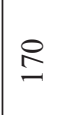 & $n$ & ' & $\sigma$ \\
\hline & 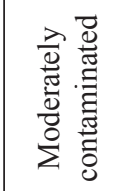 & : & in & $\stackrel{\infty}{\sim}$ & F & $\grave{o}$ & ' & $n$ \\
\hline & 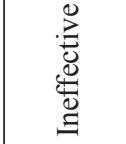 & 8 & in & $\stackrel{\infty}{\sim}$ & $\ddot{\lambda}$ & $\tilde{o}$ & ' & $\tilde{m}$ \\
\hline \multirow{3}{*}{ 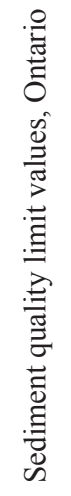 } & 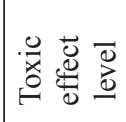 & $\underset{\infty}{ }$ & $\supseteqq$ & 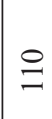 & ֶ̊ & $\circ$ & $\nabla$ & $\stackrel{n}{2}$ \\
\hline & 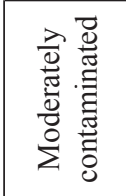 & $\stackrel{\Xi}{\beth}$ & $\stackrel{\circ}{\sim}$ & 0 & $\bar{m}$ & $\stackrel{0}{0}$ & $N$ & 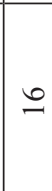 \\
\hline & 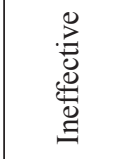 & 1 & ' & ' & ' & ' & ' & ' \\
\hline & $\begin{array}{l}\overrightarrow{\overrightarrow{0}} \\
\vec{\Xi} \\
\frac{\overrightarrow{0}}{\Delta}\end{array}$ & $\widehat{N}$ & $\ddot{U}$ & $\tilde{U}$ & $\overrightarrow{2}$ & $\tilde{U}$ & $\begin{array}{l}\stackrel{0}{0} \\
0 \\
\end{array}$ & $\bar{z}$ \\
\hline
\end{tabular}

\begin{tabular}{|c|c|c|c|c|c|c|c|c|}
\hline 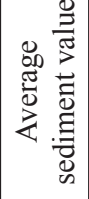 & & 6 & 亲 & f & $=$ & I & $\begin{array}{l}8 \\
8 \\
0\end{array}$ & q \\
\hline 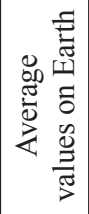 & ' & $\nabla$ & $n$ & $\approx$ & ‡ & 文 & $\begin{array}{l}8 \\
\dot{8} \\
\text { m. }\end{array}$ & ๙ิ \\
\hline 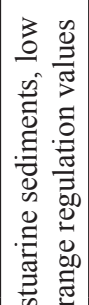 & 岌 & $\frac{\circ}{\gamma}$ & 尽 & $\stackrel{?}{i}$ & $\stackrel{\infty}{\sim}$ & $\stackrel{\circ}{\circ}$ & ' & $\frac{b}{n}$ \\
\hline 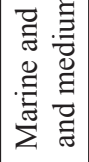 & 足 & $\stackrel{8}{n}$ & $\bar{\infty}$ & ষ্ & 它 & $\stackrel{N}{I}$ & ' & ¿े \\
\hline $\begin{array}{l}\stackrel{0}{0} \\
\stackrel{0}{\tilde{J}}\end{array}$ & $\underline{\underline{y}}$ & $\stackrel{\text { n }}{2}$ & $n$ & $\stackrel{\infty}{\sim}$ & F & $\grave{o}$ & ' & $m$ \\
\hline 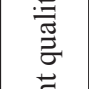 & $\overrightarrow{\mid \overrightarrow{\mid l}}$ & $\cong$ & $\stackrel{m}{m}$ & $\stackrel{\sim}{m}$ & $m$ & $\begin{array}{l}0 \\
\text { nิ } \\
0\end{array}$ & ' & $\stackrel{\infty}{\sim}$ \\
\hline 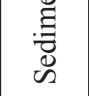 & 壬 & 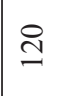 & $\stackrel{\sim}{\sim}$ & 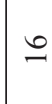 & $\bar{m}$ & $\stackrel{0}{0}$ & ' & $\underline{0}$ \\
\hline 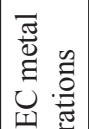 & 兽 & $\ddot{g}$ & $\exists$ & $\underset{g}{g}$ & $\stackrel{\infty}{\stackrel{\sim}{N}}$ & 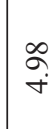 & ' & $\begin{array}{l}b \\
\infty \\
\dot{\sigma}\end{array}$ \\
\hline 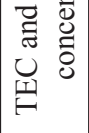 & 足 & $\vec{\beth}$ & $\begin{array}{l}\forall \\
\dot{f}\end{array}$ & $\stackrel{\circ}{\dot{m}}$ & $\begin{array}{l}\infty \\
\dot{m} \\
\text { n. }\end{array}$ & Әे. & ' & ה \\
\hline 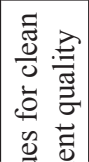 & 剀 & $\begin{array}{l}\infty \\
\dot{\oplus} \\
\dot{m}\end{array}$ & \& & $\begin{array}{l}\stackrel{0}{\circ} \\
\stackrel{0}{2}\end{array}$ & , & $\tilde{n}$ & ' & ले \\
\hline 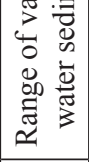 & U & $\overrightarrow{\tilde{a}}$ & $\stackrel{m}{m}$ & in & , & 光 & ' & $\stackrel{\infty}{-\infty}$ \\
\hline 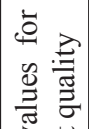 & 诳 & $\vec{\sim}$ & $\stackrel{8}{\circ}$ & $\stackrel{\infty}{\varrho}$ & $\cong$ & $\vec{\sim}$ & ' & 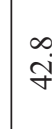 \\
\hline 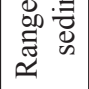 & 塤 & $\stackrel{\Xi}{\beth}$ & $\stackrel{\text { กิ }}{\text { in }}$ & $\stackrel{\infty}{\infty}$ & ֻ̃. & $\begin{array}{l}0 \\
0 \\
0 \\
0\end{array}$ & ' & $i$ \\
\hline & 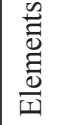 & ี & Ü & J & $\hat{\imath}$ & $\tilde{U}$ & $\begin{array}{l}\circ \\
0 \\
0\end{array}$ & $\bar{z}$ \\
\hline
\end{tabular}


Table 6. Metal concentrations determined in recent studies conducted in certain areas in Marmara region (mg/kg).

\begin{tabular}{|c|c|c|c|c|c|c|}
\hline Explanation & $\mathrm{Ni}$ & $\mathrm{Cu}$ & As & $\mathrm{Cd}$ & $\mathrm{Pb}$ & $\mathrm{Zn}$ \\
\hline $\begin{array}{l}\text { Average range in surface sedi- } \\
\text { ment }\end{array}$ & - & $77-149$ & & $0.15-0.74$ & $22-76$ & - \\
\hline Shale average value & - & 50 & & 0 & 20 & - \\
\hline 2001 & - & 417 & & 67 & 203 & - \\
\hline 2003 & - & 131 & & 2 & 81 & - \\
\hline Surface & 90 & 194 & & 6 & 510 & 890 \\
\hline Bottom & 54 & 274 & & 1 & 420 & 128 \\
\hline Halic sediment & $84-167$ & $297-3,900$ & & - & $124-702$ & $450-8,750$ \\
\hline$<63 \mu \mathrm{m}$ & - & $16-724$ & & - & $10.5-260$ & $39-793$ \\
\hline$>63 \mu \mathrm{m}$ & - & $3.5-481$ & & - & $3.13-175$ & $10-241$ \\
\hline Balat & - & 476 & & - & 95 & 614 \\
\hline Unkapani & - & 522 & & - & 152 & 585 \\
\hline Kabatas & - & 68 & & 2 & 48 & 147 \\
\hline Tarabya & - & 12 & & 2 & 6 & 19 \\
\hline R. Kavagi & - & 56 & & 1 & 17 & 82 \\
\hline Garipce & - & - & & - & - & 78 \\
\hline R. Feneri & - & - & & - & - & 120 \\
\hline Poyraz & - & - & & - & - & 40 \\
\hline Sarköy & 54 & 13 & & $<0.02$ & 23 & 44 \\
\hline M. Eregli & 21 & 30 & & $<0.02$ & 32 & 34 \\
\hline Menekse & 41 & 17 & & 1 & 22 & 50 \\
\hline Marmara & 46 & 154 & & - & 70 & 389 \\
\hline Valide Sultan Bridge & 74 & 520 & & - & 168 & 640 \\
\hline Eyup & 54 & 230 & & - & 63 & 434 \\
\hline Adalar Sonrasi & 49 & 124 & & - & 47 & 284 \\
\hline
\end{tabular}

for each metal was indicated. These values are defined by parameters such as threshold effect level, lowest effect level, minimal effect threshold level, low effect range, possible effect level, high effect level, toxic effect threshold, and medium effect range (Tables 2-3).

Tables 4-6 show the metal concentrations and their impact on the ecosystem applied in studies conducted in certain regions [8]. As a result of analysis in certain sample measurements, metal concentrations exceeded toxic effect levels when compared to sediment quality limit values and were above TEC and PEC. In analyzes of sample measurements, the metal concentration represented moderate contamination levels and remained between TEC and PEC.

The heavy metal values determined in previous studies conducted on the Marmara Region are listed in Table 6. As could be observed in these studies, the following ranges were determined as: $\mathrm{Zn}$ $10.7-8750 \mathrm{mg} / \mathrm{kg}, \quad \mathrm{Cr} \quad 4.73-485 \quad \mathrm{mg} / \mathrm{kg}, \quad \mathrm{Cu}$
3.45-3900 $\mathrm{mg} / \mathrm{kg}, \quad \mathrm{Pb} \quad 3.13-702 \quad \mathrm{mg} / \mathrm{kg}, \quad \mathrm{Cd}$ $<0.02-2.04 \mathrm{mg} / \mathrm{kg}$, Fe 5,956-55,100 $\mathrm{mg} / \mathrm{kg}$, and $\mathrm{Ni}$ $20.53-90 \mathrm{mg} / \mathrm{kg}$. When these values were compared with the results of the present study, it was observed that the heavy metal concentrations determined in this study were generally lower than the other studies in the literature.

Heavy metals that enter the marine environment due to various factors tend to accumulate in fine grain fractions of sediments. Thus, the sediments with a small diameter would result in higher heavy metal concentrations in the structure [9]. When heavy metal concentrations in the sediment samples collected from the Golden HornSutluce region were compared, we observed that the rehabilitation efforts had a positive effect. Other studies conducted in the Marmara region and the Golden Horn also demonstrated that heavy metal concentrations were higher in coastal regions in inner gulfs and inland seas when compared to high seas and middle and outer gulfs. 
Table 7. Percentages in different metal fractions (\%).

\begin{tabular}{|c|c|c|c|c|c|}
\hline Element & $\mathrm{F} 0$ & $\mathrm{~F} 1$ & $\mathrm{~F} 2$ & $\mathrm{~F} 3$ & $\mathrm{~F} 4$ \\
\hline $\mathrm{Zn}$ & $5-11$ & $7-15$ & $4-13$ & $7-14$ & $2-12$ \\
\hline $\mathrm{Cr}$ & $1-3$ & $6-16$ & $2-15$ & $8-24$ & $3-18$ \\
\hline $\mathrm{Cu}$ & $2-6$ & $6-16$ & $4-12$ & $6-14$ & $2-17$ \\
\hline $\mathrm{Pb}$ & $2-4$ & $7-9$ & $5-11$ & $2-10$ & $2-11$ \\
\hline $\mathrm{Cd}$ & $3-5$ & $5-7$ & $4-8$ & $2-12$ & $3-9$ \\
\hline $\mathrm{Ni}$ & $6-9$ & $5-8$ & $5-12$ & $12-16$ & $3-13$ \\
\hline
\end{tabular}

Analysis results demonstrated that metal concentrations exceeded TEC values and/or were between TEC and PEC. Concentrations between TEC and PEC values are also considered to have poor effects in certain cases. Other values were below TEC. Under TEC, it is accepted that adverse effects were observed only rarely. The metal concentrations obtained in the present study were ordered by the heavy metal content in the sediment as follows: $\mathrm{Zn}>\mathrm{Cu}>\mathrm{Cr}>\mathrm{Pb}>\mathrm{Ni}>\mathrm{Cd}$.

Although the metals may remain fixed in the sediment for a long time, they could be freed by degradation under oxidizing conditions. This situation is explained by the presence of metal fractions. Heavy metals are separated into fractions based on their reaction with soil particles due to environmental conditions. In sequential metal extractions on sediment samples, F0 represents the fraction of water-soluble metals, F1 represents the fraction of exchangeable metals, F2 represents the fraction of carbonate-bound metals, F3 represents the fraction of Fe-Mn oxide-bound metals, F4 represents the fraction of organic matter and sulfide-bound metals, and $\mathrm{F} 5$ represents the fraction of metals in the residual section.

The $\mathrm{Cr}, \mathrm{Cu}, \mathrm{Cd}, \mathrm{Ni}, \mathrm{Pb}$, and $\mathrm{Zn}$ concentrations in different samples and rate ranges found in different fractions in the total concentrations are presented in Table 7. Chemical fractions distinguish metals of natural origin and/or metals of anthropogenic sources. Metal fractionation possesses a critical significance for potential toxicity and mobility of metals [10]. The sequential extraction technique was introduced for this purpose to provide information on the strength and forms of the metals associated with the sediments [11]. According to the analysis results, it could be clearly observed that the distribution of heavy metal fractions in different areas in the sediment varied significantly due to various factors.

As seen in the analysis results, $\mathrm{Cr}, \mathrm{Cu}, \mathrm{Cd}, \mathrm{Ni}, \mathrm{Pb}$, and $\mathrm{Zn}$ were mostly found in the residual fractions of the samples, indicating that these metals were bound to mineral mesh in the samples. The highest concentrations after the residual fractions were found in reducible fractions (i.e., metals were bound to Fe and Mn oxides).

The $\mathrm{Zn}$ concentrations were predominantly in persistent/bound fractions (mean 50\%) and the average $\mathrm{Zn}$ concentrations in the exchangeable, reducible fractions were $20 \%$ of the total concentration. It was observed that
$\mathrm{Zn}$ in the sediment found in this study region might be detrimental to biota in the aquatic environment, since it could be re-mobilized when environmental conditions change. The average $\mathrm{Zn}$ concentration in oxidizable fractions was about $10 \%$ of the mean total concentration.

High amounts of $\mathrm{Cr}$ and $\mathrm{Cu}$ were found in residual fractions, while large rates $(18 \% \mathrm{Cr}$ and $11 \% \mathrm{Cu})$ were found in reducible fractions. Fraction F3 (associated with $\mathrm{Fe}$ and $\mathrm{Mn}$ oxides) was defined as the reducible fraction, and metals in this fraction could be released if exposed to sediment reduction conditions according to the information available in the literature [12-13]. Fe (II) and Mn (II), Fe (III) and Mn (IV) are oxidized to form oxide or hydroxide precipitates in increasing oxygen concentrations $(\mathrm{Eh}>100 \mathrm{mV})$ and neutral $\mathrm{pH}$ media. Several metal types can be adsorbed on this type of precipitate.

The non-fixed $\mathrm{Cr}, \mathrm{Cu}$, and $\mathrm{Zn}$ fractions (F1, F2, F3) were higher than the fixed fractions. Fixed $\mathrm{Pb}$ and $\mathrm{Ni}$ concentrations were also relatively high. This demonstrated that the natural structure could contribute to certain $\mathrm{Pb}$ values in the sediment in the study area. Ni was probably immobilized in aluminosilicate minerals and might be found in biologically negligible amounts [14-15]. Ni and $\mathrm{Zn}$ concentrations in highly variableexchangeable fractions could be easily absorbed and utilized by the organisms.

Only small amounts ( $<9 \%$ of total concentrations) of $\mathrm{Cr}, \mathrm{Cu}, \mathrm{Ni}$, and $\mathrm{Pb}$ were found in the exchangeable fractions in the sediment samples, indicating that these metals would not pose a significant risk to aquatic biota because only small amounts could be reactivated when environmental conditions change [16]. The multitude of exchangeable fractions suggested that anthropogenic activities (mainly due to the discharge of sewer and industrial wastewater into the streams) affected the metal concentrations in the study area $[17,18]$.

\section{Conclusions}

In the analyses, the concentrations of human activity-originated $\mathrm{Zn}, \mathrm{Cr}, \mathrm{Cu}, \mathrm{Pb}, \mathrm{Cd}$, and $\mathrm{Ni}$ heavy metals were determined from sediment, and mean heavy metal concentrations were found as follows: $\mathrm{Zn}$ 70-260, Cr 26-110, Cu 70-135, Pb 8-50, Cd 0.1-2, and Ni $10-38 \mathrm{mg} / \mathrm{kg}$. Concentrations were usually low and indicated that the sediment was not too polluted in the present. When the values obtained in the study were compared with the literature, we could conclude that the bed dredging works in the Golden Horn, the channels established around the estuary to prevent wastewater discharge, building of wastewater treatment plants, and the closure of the existing factories improved the rehabilitation of heavy metal levels in the sediment. However, contaminants that were concentrated in the sediment could persist in the sediment structure in the Golden Horn, even if the pollutant discharge to the water environment is reduced or even prevented. 
Since the Golden Horn has been exposed to heavy metal pollution during the past, it would take a long time to clean up the pollution in the sediment and to reduce the heavy metal pollution in the living organisms inhabiting this area, even if all the pollution sources are restricted. To prevent pollution in the Golden Horn, all uncontrolled discharges from land, air and water sources should be identified and prevented. Industrial organizations located around the Golden Horn should be prevented from conducting illegal discharges by regular inspections, and treatment plant effluents complying with the discharge standards should be controlled.

\section{Acknowledgements}

This research has been supported by Yildiz Technical University Scientific Research Projects Coordination Department (Project Number: 2014-05-02-KAP02). We would like to express our gratitude to Fatma Kazak for helping the analysis.

\section{References}

1. COLEMAN H.M., KANAT G., TURKDOGAN İ. Restoration of the Golden Horn Estuary (Halic). Water Research, 43 (20), 4989, 2009.

2. AYDIN Z.S. The Characteristics and Refuse of Golden Horn Surface Sediment. Master Thesis, Bogazici University. 1994

3. TUNCER G., TUNCEL G., BALKAS T.I. Evolution of Metal Pollution in the Golden Horn (Turkey) Sediments Between 1912 and 1987. Marine Pollution Bulletin, 42 (5), 350-360. 2001.

4. IBB. (Istanbul Büyüksehir Belediyesi), Golden Horn Water Basin- Halic Havzasi, Available online: http://katalog.ibb. gov.tr/kutuphane2/YordamVt/projem_istanbul/pi_00038. pdf. (Accessed on 27 June 2015).

5. KAHVECIOGLU O., KARTAL G., GUVEN A., TIMUR S. Environmental Effects of Metals-I, TMMOB Metalurji Mühendisleri Odası Metalurji Dergisi, 136, 47, Available online: http://www.metalurji.org.tr/dergi/dergi136/ d136_4753.pdf. (Accessed on 20 May 2015) [In Turkish].

6. KONCAVAR M. Istanbul's Shipyards- İstanbul'un Tersaneleri, TMH (Türkiye Mühendislik Haberleri) Kentleşme ve İstanbul, 413, 40, 2001 [In Turkish].
7. APHA. Standard methods for the examination of water and wastewater, Washington, DC. 2005.

8. CUBBAGE J., BATTS D. Summary of Guidelines for Contaminated Freshwater Sediments. Available online: https://fortress.wa.gov/ecy/publications/publications/95308. pdf. (Accessed on 2 February 2015).

9. SINGH, A., HASNAIN, S. \& BANERJEE, D. Grain size and geochemical partitioning of heavy metals in sediments of the Damodar River - a tributary of the lower Ganga, India. Environmental Geology, 39 (1), 90, 1999.

10. MAIZ I., ARAMBARRI I., GARCIA R., MILLAN E. Evaluation of heavy metal availability in polluted soils by two sequential extraction procedures using factor analysis. Environmental Pollution 110 (1), 3, 2000.

11. TESSIER A., CAMPELL P.G.C., BISSON M. Sequential extraction procedure for the speciation of partition of particulate trace metals. Analytical Chemistry, 51 (7), 844, 1979.

12. SIMPSON S.L., ANGEL B.M., JOLLEY D.F. Metal equilibration in laboratory-contaminated (spiked) sediments used for the development whole-sediment toxicity tests. Chemosphere, 54 (5), 597, 2004.

13. BORCH T., KRETZSCHMAR R., KAPPLER A., VAN CAPPELEN P., GINDER-VOGEL M., VOEGELIN A., CAMPBELL K. Biogeochemical redox processes and their impact on contaminant dynamics. Environmental Science and Technology, 44 (1), 15, 2010.

14. NEMATI K., BAKAR N.K.A., ABAS M.R. Speciation of heavy metals by modified BCR sequential extraction procedure in different depths of sediments from Sungai Buloh, Selangor, Malaysia. Journal of Hazardous Materials, 192 (1), 402, 2011.

15. SUNDARAY S.K., NAYAK B.B., LIN S., BHATTA D. Geochemical speciation and risk assessment of heavy metals in the river estuarine sediments-A case study: Mahanadi basin, India. Journal of Hazardous Materials, 186, 1837, 2011.

16. LI G.G., HU B.Q., BI J.Q., LENG Q.N., XIAO C.Q., YANG Z.C. Heavy metals distribution and contamination in surface sediments of the coastal Shandong Peninsula (Yellow Sea). Marine Pollution Bulletin, 76 (1), 420, 2013.

17. SUTHAR S., NEMA A.K., CHABUKDHARA M., GUPTA S.K. Assessment of metals in water and sediments of Hindon River, India: impact of industrial and urban discharges. Journal of Hazardous Materials, 171 (1-3), 1088, 2009.

18. XIAO R., BAI J., HUANG L., LIU X. Distribution and pollution, toxicity and risk assessment of heavy metals in sediments from urban and rural rivers of the Pearl River delta in southern China. Ecotoxicology, 22, 1564, 2013. 
